\title{
Zur Funktion der aristotelischen Ursachenlehre in der Scholastik
}

\author{
ROLF SCHÖNBERGER (München)
}

Jene Bemühung der Griechen, in der Philosophie und Wissenschaft noch ungeschieden sind, die sich aber grundsätzlich und wirksam dem Mythos entgegensetzt, jene Bemühung beginnt mit der Suche nach Gründen. War der Mythos zwar ebenfalls eine Form, das Geschehen im Naturablauf hinsichtlich seiner auffälligsten Phänomene zu verstehen, so setzt sich jetzt gegen ihn der Versuch ab, diese Phänomene und die begegnende Welt insgesamt durch ihre Gründe begreiflich werden zu lassen. Diese Bemühung initiiert zum einen eine neue Mentalität, wie sie etwa in dem Satz des Demokrit zum Ausdruck kommt: „er wolle lieber einen einzigen ursächlichen Beweis [aitiologia] für etwas finden, als daß er den persischen Königsthron erwürbe “1. Aus der Perspektive des Anspruches, für vertretene Behauptungen einen Grund abfordern zu können bzw. angeben zu müssen, rücken alle bisherigen Formen existentieller Verläßlichkeit wie Mythos und Nomos auf die Seite der doxa. Da jenes Wissenwollen aber nicht bloß die überkommenen Strukturen der Verläßlichkeit auf neuen Feldern anwendet, sondern insgesamt relativiert, verlangt sie zum anderen -- also über die eben genannte Verwandlung der Mentalität hinaus auch eine eigene theoretische Fundierung: Gibt es denn so etwas wie Gründe, und wenn ja, wofür überhaupt lassen sich Gründe angeben? Zunächst sieht es so aus, als ob sich dies aus der Art des theoretischen Umgangs selbst ergäbe, in dem nichts aus der Warum-Frage ausgespart wird: weder die Erscheinungen der Natur noch die Gehalte des Nomos oder die Herkunft der Götter, weder das Erstaunliche noch das Selbstverständliche.

Die diversen philosophischen Ansätze der Antike ließen sich wohl ohne jede verfremdende Äußerlichkeit nach den Formen der in ihrer Suche

\footnotetext{
1 Frg.118; Übersetzung von H.-G. Gadamer, Antike Atomtheorie (1935), in: Gesammelte
} Werke V, Tübingen 1985, 271 sq. 
unterstellten Gründe gruppieren. Platon hat in einer berühmten Passage des „Phaidon“ dies in Form der sokratischen Biographie geschildert. Die dort vorgetragene Kritik Platons an Anaxagoras hat Leibniz übersetzt, um sie in den „Discours de métaphysique“ (im Kapitel 20) einzufügen (Phaid. $97 \mathrm{c})^{2}$. Ungeachtet dieser ersten Auseinandersetzung um die Arten (eide) von aitiai und ungeachtet der inhaltlichen Kontroversen darüber, was wofür Ursache ist, bleibt dabei doch gemeinsame Voraussetzung, daß es als unproblematisch gelten kann, Gründe überhaupt zu suchen. Von Aristoteles bis Thomas Hobbes wird denn auch immer wieder die Philosophie geradezu als Suche nach Gründen definiert.

Gleichwohl rückt spätestens mit Platon die Frage in den Mittelpunkt, welche Arten von Gründen es denn „gibt“. Was bei Platon als philosophische Entwicklung des Sokrates geschildert wird, erhält bei Aristoteles die Form einer geschichtlich sukzessiven Entdeckung der verschiedenen Typen des Grund-seins, die - aristotelischem Selbstverständnis nach bei ihm selbst zum Abschluß kommt.

Im übrigen sei gleich vorweg bemerkt: Wenn im folgenden auch weiterhin von den „vier Ursachen“ die Rede ist, so geschieht dies nur faute de mieux. Der moderne Begriff der Ursache macht es ziemlich problematisch, mit Bezug auf ein Ding oder ein Ereignis von mehreren Ursachen zu sprechen. Die deutsche Sprache verfügt jedoch über kein Substantiv mit ähnlich breiter Bedeutung wie das griechische aitia ${ }^{3}$.

Es mag vielleicht zu den Rätseln der Denkgeschichte gehören, daß ein in fundamentalen Fragen - wie etwa im Konzept der Metaphysik, der Vollendung des Menschen oder der Theorie und Pragmatie des Wissens - zweideutig bleibender Autor wie eben Aristoteles zu einem Schulautor werden konnte, doch scheint das Lehrstück von den vier Ursachen ein vergleichsweise eindeutig fixiertes zu sein, auf das man sich nur zu berufen braucht. Unklar ist allenfalls seine theoretische Genese. Denn Aristoteles präsentiert das Theorem nur im Resultat. Ebendies aber hat seine Exegeten irritiert. Sir David Ross schreibt in seinem Kommentar zur Physik: „We do not know how Aristotle arrived at the doctrine of the four causes; where we find the doctrine in him, we find it not argued for but presented as self-evident" ${ }^{\text {" }}$. Diese Verwunderung ist für Wolfgang Wieland in seinem inspirativen Buch über "Die aristotelische Physik“ bereits ein Indiz dafür, daß Ross hier noch in einer Auslegungstradition stehe, die mit überstrengen systematischen Ansprüchen an die aristoteli-

2 Ed. C. J. Gerhardt, Die philosophischen Schriften von G. W. Leibniz, ND Hildesheim (Olms) 1960, VII, 335.

3 Heidegger, Nietzsches Lehre vom Willen zur Macht als Erkenntnis, GA XLVII, 184: "Griechisch gedacht, meint aîtıov jenes, woran es liegt, daß ..."

4 Aristotle's Physics. A Revised Text with Introduction and Commentary, London 1936, 37. 
schen Texte herangehe. „In Wirklichkeit“, sagt Wieland, „,handelt es sich bei der Vierursachenlehre aber gar nicht um eine hintergründige Theorie von metaphysischen Grundprinzipien, die durch eine glückliche Fügung der Natur dem menschlichen Geist als selbstevident gegeben sind und unmittelbar einleuchten, sondern um etwas viel Einfacheres ...[:] um das Ergebnis einer Analyse des Sprachgebrauchs“"5. Hans Wagner hat sich trotz seiner ansonsten scharfen Kritik an Wieland dieser Sicht der Dinge im wesentlichen angeschlossen. Der „Ausgangspunkt der gesamten Gründelehre“, sagt Wagner in seinem Physik-Kommentar, sei „die Analyse der Warum-Antworten" "6; daher könne Aristoteles zum einen als Grund auch Negativität anführen und zum anderen ohne weiteres für dasselbe mehrere Gründe angeben.

Gleichwohl ist es doch ebensowenig unproblematisch, daß Aristoteles dieses Lehrstück nicht allein an zwei verschiedenen Stellen vorträgt, sondern auch, daß diese Passagen in der Metaphysik und in der Physik dem Gehalt nach sich nahezu unverändert finden. Man muß fragen: 1. In welches der beiden Gebiete gehört denn diese Theorie von den sog. vier Ursachen? 2. Wodurch unterscheiden sich Physik und Metaphysik, wenn nicht hinsichtlich der jeweils gesuchten Arten von Gründen? Immerhin könnte es doch naheliegend sein, wenn Wissen heißt, Gründe anzugeben vermögen, daß dann die verschiedenen Arten des Wissens durch verschiedene Arten von Gründen definiert sind.

Wieland hat diesen Unterschied für irrelevant erachtet. Die Physik sei „aus sich selbst verständlich“7 und zudem in heutiger Terminologie ,der

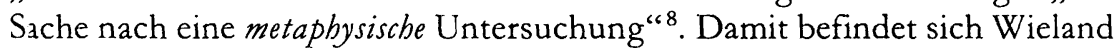
auch selbst schon in einer, wenn auch nicht weiter namhaft gemachten Tradition. Ebenso wie nach Hegel die aristotelische Physik das „ist, was fur die jetzigen Physiker eigentlich die Metaphysik der Natur wäre"99, hat auch Heidegger in seinem Aufsatz „Vom Wesen und Begriff der Physis“ k)nstatiert: „überhaupt hat es wenig Sinn zu sagen, die ,Physik“ gehe der

Die aristotelische Physik, Göttingen 1962, 262. Ganz ähnlich die Stoßrichtung von Heidegger, Die Frage nach der Technik [1953], in: Vorträge und Aufsätze, Pfullingen 51985, 12: „Zwar tut man seit Jahrhunderten so, als sei die Lehre von den vier Ursachen wie eine sonnenklare Wahrheit vom Himmel gefallen. Indessen dürfte es an der Zeit sein zu fragen: weshalb gibt es gerade vier Ursachen? Was heißt in Bezug auf die genannten vier eigentlich, Ursache?? Woher bestimmt sich der Ursachecharakter der vier Ursachen so einheitlich, daß sie zusammengehören?"

Aristoteles, Physikvorlesung, Darmstadt ${ }^{4} 1983,463$ (40,1-7); ebenso W. Charlton, Aristotle's Physics I, II. Translated with Introduction and Notes, Oxford ${ }^{2} 1983,99$; kritisch dazu jüngst M. Schofield, Explanatory Projects in Physics, 2.3 and 7, in: Oxford Studies in Ancient Philosophy, suppl. vol.: Aristotie and the Later Tradition, ed. H. Blumenthal and H. Robinson, $29-40$.

Op. cit., 13.

Op. cit., 19; cf. p. 60 sq.

Vorlesungen über die Geschichte der Philosophie, XVIII (Glockner), 337. 
,Metaphysik' voraus, da die Metaphysik ebenso sehr ,Physik' ist als die Physik ,Metaphysik“"،10.

Wie immer, es steht gleichwohl fest, daß es sich nach aristotelischem Selbstverständnis ganz ohne Zweifel um zwei voneinander zu unterscheidende Disziplinen handelt. Die Weise, wie Aristoteles in Metaphysik E 2 die Unterscheidung zwischen Physik und Metaphysik angibt, verbleibt nun aber gänzlich innerhalb der Lehre von den Substanztypen. Die Disziplinen werden dabei nicht neuzeitlich methodisch, sondern inhaltlich gegliedert. Auf der Basis zweier Kriterien, nämlich der Selbständigkeit und des Bewegtseins ergeben sich durch jeweilige Kontradiktion drei relevante Kombinationen, denen die drei theoretischen Disziplinen zugeordnet werden ${ }^{11}$. Die Einordnung der Ursachenlehre bleibt von daher gesehen also weiterhin offen und problematisch.

II.

Jene drei hier nur gestreiften Interpretationsprobleme - nämlich erstens die wissenschaftstheoretische Zuordnung der Ursachenlehre, zweitens die Frage der möglichen aitiologischen Unterschiedenheit von Naturphilosophie und Metaphysik, und drittens das Problem ihrer theoretischen Genese -, diese drei Interpretationsprobleme wachsen im Aristotelismus, soll heißen im Verlauf der Interpretationsgeschichte, zusammen. Im folgenden sei nun ein Blick darauf geworfen, wie gerade im Ausgang von der aristotelischen Ursachenlehre selbst eine Unterscheidung von Physik und Metaphysik konzipiert wird. Dabei wird sich zeigen, daß entweder den beiden Disziplinen unterschiedliche causae zugeordnet oder diese causae, um als Unterscheidungskriterium fungieren zu können, in sich differenziert werden. In beiden Fällen aber geschieht auf der Basis des Aristoteles zugleich ein deutlicher Schritt über ihn hinaus.

Die Frage muß ansetzen beim inneren Zusammenhang der vier Ursachen oder noch eher dabei, ob ein solcher Zusammenhang überhaupt besteht. Sollten diese vier Ursachen ebenfalls nur, wie es Kant von den Kategorien des Aristoteles behauptet hat, "rhapsodistisch“ aufgelesen (KrV B 106) sein oder sind sie in ihrem Erschöpfendsein von einem Prinzip her einsichtig zu machen? Solche Systematisierungsversuche können - gegen Wieland - nicht als dem Aristoteles völlig äußerlich angesehen werden:

${ }^{10}$ GA IX, 242; cf. Einführung in die Metaphysik, Tübingen ${ }^{4} 1976,14$ (= GA XI., 20): „Die ,Physik' bestimmt von Anfang an das Wesen und die Geschichte der Metaphysik ..."; cf. 107 (=GA XL, 149); Der Satz vom Grund, Tübingen ${ }^{5} 1978,120$.

1 Prägnant zusammengefaßt bei E. Vollrath, Aristoteles: Das Problem der Substanz, in: J. Speck (ed.), Grundprobleme der großen Philosophen, Göttingen ${ }^{2} 1978,84-128$; hier $93-96$. 
Zum einen hat Aristoteles auch seinerseits mitunter solche Suffizienzanalysen angestellt, etwa bei der Zahl der Sinne (de an. III, 1); zum anderen kommt er immer wieder in seinen Schriften auf die Vierzahl der Ursachen als einer festen Größe zu sprechen und schließlich hat der problemgeschichtliche Exkurs in Metaphysik A 3-7 eben vor allem den Sinn, die Vollständigkeit der vier Ursachen abzusichern ${ }^{12}$.

Für eine Denkform wie die der Scholastik ist es nun aber geradezu typisch, die Frage aufzugreifen, wie sich die Suffizienz der sog. vier Ursachen theoretisch zwingend zeigen ließe. Innerhalb der lateinischen Scholastik hat Thomas von Aquin das Suffizienzproblem der vier Ursachen bereits in seinen frühen Schriften erörtert ${ }^{13}$. Eine für unsere Frage relevante Bedeutung bekommt es aber, so scheint es, erst bei Dietrich von Freiberg. Während es nämlich sonst in vielen, auch späteren Physik- und MetaphysikKommentaren übergangen wird ${ }^{14}$, greift Dietrich immer wieder dies Problem auf.

In der Schrift „De animatione caeli“ führt Dietrich die vier Ursachen präzise als vier Gattungen von Ursachen an ${ }^{15}$. Von Anfang an werden sie mit Averroes so angesetzt, daß sie als ganze im eigentlichen Sinne nur in demjenigen Bereich von Wirklichkeit vorkommen, der als bewegter der Veränderung, dem Werden und Vergehen unterworfen ist. Schon auf der Stufe der Mathematik aber ergibt der Begriff des Zweckes keinen Sinn; nichts ist mathematisch deswegen der Fall, weil es so gut ist ${ }^{16}$; wenn aber - so muß man ergänzen - der Zweck die Kausalität der Wirkursache bestimmt, dann kann man mit Dietrich sagen, daß dann per consequens (!) auch die Wirkursache dort keine Rolle spielen kann. Gilt dies bereits in der Mathematik, dann ist es bei den materie- und deshalb bewegungsfreien Substanzen nur umso plausibler ${ }^{17}$. Die Metaphysik schließt also die causa

12 Heidegger, der sonst vor solchen Deduktionsansprüchen bei der aristotelischen Kategorienlehre gewarnt hat (Nietzsche: Der europäische Nihilismus, GA XLVIII, 68), bringt im „Vom Wesen des Grundes“ (GA IX, 124 sq.) genau das zuletzt genannte Argument vor. Allerdings urteilt auch er später hier anders, Die Metaphysik des deutschen Idealis-

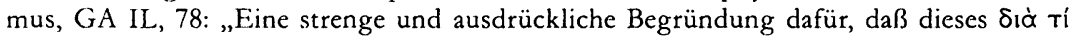
oder die aitía vierfach ist, gibt Aristoteles in den uns erhaltenen Schriften nirgends."

13 De princ. naturae, cap. 4 (ed. Leon. XLIII p. 43 sq.); Albertus Magnus, Phys. II, 7 (ed. Col.IV 1, 107 sq.) - Beide ordnen die Frage nach den Ursachen als solchen der Ersten Philosophie zu: Thomas, in Phys. II, 5 (176); Albert, op. cit., 106, 69-72; 108, 11-15; cf. Heinrich von Gent (?), Quaestiones in librum de causis, Resp. ad 3 (ed. J. Zwaenepoel 25).

14 Die Vierzahl nur feststellend und erläuternd: Roger Bacon, comm. natural. I p.II. d.5 c.1 (ed. R. Steele, Opera hactenus inedita, Oxford 1905 sqq., 121-124; in den PhysikQuaestiones des Boethius von Dacien (CPDMA V, 2) fehlt die Fragestellung ganz; in späteren Texten wie den Physik- und Metaphysik-Quaestiones des Johannes von Jandun verlagert sich die Frage auf die Wirkweise der causa finalis.

15 So auch de int. II 8, 1 (I, 151): per singula causarum genera.

16 Cf. Aristoteles, Met. III, 2; 996a35-bl; allerdings Thomas, in Met. III, 4 (385).

17 De anim. caeli 3, 2 (III, 14 sq.); de orig.1, 8 (III, 139); de luce 1, 3 (IV, 9). 
finalis und die causa efficiens aus ihrer Betrachtungsweise aus; sie ist Metaphysik durch diesen Ausschluß.

Für unseren Zusammenhang ist jedoch jetzt von gleichem Gewicht, daß Dietrich innerhalb der vier Ursachengattungen einen doppelten ordo essentialis dependentiae ansetzt. Die vier Gattungen von Ursachen hängen voneinander wesentlich ab: Die Materie steht, wie Dietrich sagt, unter der Ordnung der Form; die Form nämlich prägt $(\text { figit })^{18}$ die Materie ins Sein. Die Form wiederum steht unter der Ordnung des agens, welches die Form ins Sein überführt (deducere). Das agens wird aber nun seinerseits zum Agieren vom Zweck bewegt. Genauer und noch vollkommener muß jedoch nach Dietrich folgendermaßen gesagt werden: Die Materie steht unter der Ordnung der Form und diese beiden, Form und Materie, stehen unter der Ordnung des agens und diese drei insgesamt zuletzt unter der Ordnung des finis. Der Zweck ist daher in dem Sinne die erste der Ursachen, als er causa causalitatis ist ${ }^{19}$.

Nur deshalb, weil hier eine wesentliche Abhängigkeit unter den Gattungen von Ursachen besteht, kann erstens ausgeschlossen werden, daß sich die Dinge nur äußerlich und zufällig auseinander ergeben und zweitens, daß einzelne Ursachtypen aus diesem Geflecht von Abhängigkeiten wieder herausgebrochen werden.

Die zweite Form wesentlicher Abhängigkeit findet sich nach Dietrich innerhalb der einzelnen Gattungen von Ursachen; das gehört nicht unmittelbar zu unserem Thema. Wichtiger ist nämlich die Frage, welche kausalen Verhältnisse überhaupt bei materiefreien Substanzen herrschen. Dietrich sagt nun, daß die zwei Typen der äußeren Ursachen, also agens und finis, ihrem eigentlichen Sinngehalt nach dort ausgeschlossen sind. Eine Wirkursache oder ein Zweck bilden keine Gründe, welche die Metaphysik angibt. Die anderen beiden Gründe werden der Physik zugeordnet. Immerhin darf nicht übersehen werden, daß Dietrich für Effiziens und Finalität konzediert, daß sie bei den unbewegten Substanzen vorkommen secundum quandam proportionem ${ }^{20}$. Er nennt denn auch ausdrücklich das Motiv dieser Konzession: Ohne eine zumindest analoge Realisierungsweise

${ }^{18}$ Ein im übrigen häufig benutztes Wort Dietrichs, auch im Liber de causis des öfteren zu finden; cf. Eckhart, Eccl.n.45 (LW II, 274). - Zur causa essentialis: B. Mojsisch, Meister Eckhart. Analogie, Univozität und Einheit, Hamburg 1983, 24-29; id., ,Causa essentialis‘ bei Dietrich von Freiberg und Meister Eckhart, in: Von Meister Dietrich zu Meister Eckhart, ed. K. Flasch, Hamburg 1984, $106-114$.

19 De anim. caeli 4, 2 (III, 15): das Voraussetzungsverhältnis der vier causae auch in de cogn. ent. sep. 32, 2 (II, 195 sq.); ähnliche Formulierungen auch anderwärts: Alexander von Hales, sum.theol. I n.104 (I, 183 b); Thomas von Aquin, de princ.nat.4 (ed. Leon.XLIII p. 44, 1.35-36): ,finis est causa causarum, quia est causa causalitatis in omnibus causis"; Philipp der Kanzler, Summa de bono, q. 1 (ed. N. Wicki, Bern 1985, I, 6); Siger v. Brabant, qu.in Phys. II q. 17 (ed. Bazán 177); Duns Scotus, ord.I d.2 n.89 (ed.Vat II, 180); zurückgehend wohl auf Avicenna, Met.VI, 5 (Van Riet, 338).

${ }^{20}$ De anim. 5, 2 (III, 16). 
der Ursachen wäre die Einheit des Kosmos nicht zu denken. Es ist aber auch offenkundig, daß ohne diese Konzession jene Deduktion der Ursachearten nachträglich wieder dementiert würde.

Wenn Analogie keine Ausflucht sein soll, dann muß entweder die Differenz angegeben oder doch begründet werden, warum sie nicht im einzelnen angebbar ist. Dietrich setzt der Transmutationskausalität ein einfaches Hervorgehen entgegen. Obwohl in seinen Schriften der Schöpfungsgedanke mit Ausnahme der Bemühung, das voraussetzungslose Hervorgehenlassen der immateriellen Substanzen nicht mit dem primum principium koinzidieren zu lassen ${ }^{21}$, keine sonderliche Rolle spielt, ist doch offenkundig, daß Dietrich sich hiermit einem Konzept anschließt, das auch die creatio noch als ein ursächliches Verhältnis denken wollte. Bei der Unterscheidung von drei Weisen des ursächlichen Hervorgehens (causalium processuum) charakterisiert Dietrich die erste Weise folgendermaßen: „entia per suas essentias emanent modo simplici ab aliquo principio omnino separato ...; et dico ,modo simplici', quia non per motum vel quamcumque transmutationem, sed per simplicem defluxum totalis essentiae rei sic emanantis" 22 . Eine sehr ähnliche Entgegensetzung bringt übrigens auch bereits Thomas von Aquin an einer besonders signifikanten Stelle in seinem Physik-Kommentar: „productio universalis entis a Deo non sit motus nec mutatio, sed sit quaedam simplex emanatio" 23 . In beiden Fällen wird die Ebene der Bewegung überschritten. Weder der Hervorgang des Intellektes bei Dietrich noch die Mitteilung von Sein in der ontologischen Metaphysik kann als Transmutation gedacht werden. Aber auch Siger von Brabant und andere kennen diese Unter-

${ }^{21}$ De subst. spir. 19, 1 (II, 316).

22 De anim. 7, 1 (III, 17). K. Flasch, Einl. op. omn. III p. XXX: „die effiziente Kausalität und die finale finde sich ausschließlich im Bereich der äußeren Natur“; „metaphysische Begründungsverhältnisse“ würden nicht „kritiklos nach dem Modell effizienter Kausalität" (ibid.) ausgelegt; in der "Analyse der Hauptformen des Begründens" ergebe sich, „daß die effiziente Kausalität ein Extremfall von Äußerlichkeit und Zufälligkeit" (ibid.) darstelle; cf. XXXII sq. Man fragt sich, aus welcher Perspektive sich diese Einschätzung ergibt: Inwiefern etwas als zufällig gelten kann, ist ja so simpel nicht (cf. A. Maier, Notwendigkeit, Kontingenz und Zufall, in: Die Vorläufer Galileis im 14. Jahrhundert [Studien zur Naturphilosophie der Spätscholastik I], Rom 1949, 219-250; Thomas, Sum. theol.I, 82, 1); die Entgegensetzung von Bewegungskausalität und einfacher emanatio ist zudem durchaus gängig.

23 In Phys. VIII, 2 (974); Sent.I d.7, 1, 1 ad 3: "agens enim naturale est causa motus; sed agens divinum est dans esse totum"; für die zwei Weisen, wie etwas als agens auftreten kann, beruft sich Thomas in Sent. II d.1, 1, 2 ad 4 auf Avicenna, Suff.I, 10 und Met. VI, 1 (Van Riet 292). Bemerkenswert ist etwa auch die schrittweise Umkehrung der aristotelischen Behauptung, der Begriff der Möglichkeit habe zwar im Kontext der Bewegung seine „eigentlichste“ Bedeutung, diese sei aber in der metaphysischen Ausweitung auch auf das Nicht-Bewegte nicht die ,dienstlichste“ (Met. $\Theta, 1 ; 1045 \mathrm{b36}$ ). Thomas hält in seinem Kommentar dazu (Met. IX, 1; nr. 1170-1772) den Ausgang vom Bewegungsphänomen deshalb für nützlich, weil dieses uns näher ist, die eigentliche Absicht gehe aber auf die Bedeutung von Akt und Potenz beim Seienden ganz allgemein. 
scheidung. Bei der Frage, ob ewig Dauerndes eine Ursache habe, macht Siger folgende Unterscheidung: Eine Ursache, die durch Veränderung ihre Wirkung hervorbringt, kommt nicht in Frage; nur ist damit nicht der ganze Sinn des Begriffes erschöpft: „Alio potest intelligi aliquid habere causam efficientem per hoc quod sit causa suae naturae et sui esse dans sibi esse, sic quod non est causa esse vel fieri per transmutationem; et tale non tollit rationem effectus. Et sic possunt sempiterna babere causam sui esse" ${ }^{24}$. Aegidius Romanus scheint, um diesen Sachverhalt ausdrücken zu können, den Terminus essefactio geprägt zu haben.

III.

Als erster hat $\mathrm{m}$. W. Kurt Flasch auf die Übernahme averroistischer Elemente in Dietrichs Kausalitätskonzeption aufmerksam gemacht. Im Blick auf dessen Unterscheidung der Metaphysik von der Logik nannte er Dietrichs „Abgrenzung von der Physik“ eine sehr viel „deutlichere Neuerung" ${ }^{25}$. Und schon in der Einleitung zum ersten Band der Opera omnia Dietrichs hatte er auf die entsprechende Übernahme dieses Gedankens bei Meister Eckhart ${ }^{26}$ hingewiesen und diese Übernahme als einen „Affront gegen den aristotelischen und thomistischen Metaphysikbegriff ${ }^{\prime 27}$ bezeichnet.

Ohne dies im einzelnen diskutieren zu wollen möchte ich vielmehr drei in sich sehr unterschiedliche Ergänzungen hinzufügen; die erste betrifft den Versuch, noch etwas in der Vorgeschichte dieses Gedankens zu sondieren; die zweite betrifft einige Variationen der Rezeptionsgeschichte; die dritte betrifft einen Hinweis auf eine formal gesehen ähnliche, inhaltlich aber nahezu spiegelverkehrte Konzeption.

1. Der Versuch, die Neuerung in ihrer Tragweite genauer zu bestimmen, wird sich zunächst an die einschlägigen Kommentare wenden. Albertus Magnus etwa sagt in seinem Physik-Kommentar (aus den frühen $1250 \mathrm{cr}$

${ }^{24}$ Met. II q. 8 (ed. Dunphy 60). Ebenso kennt Siger die Unterscheidung von causa efficiens in esse et in fieri III q. 3 (Dunphy 93) sowie die Formel unde principium esse - unde principium motus III q. 4 (ed. Dunphy 95), eine grundsätzliche wissenschaftstheoretische Konsequenz zieht er daraus aber nicht. - Zu Meister Eckhart, Eccl.48 (LW II, 276); cf. infra n.33.

${ }_{25}$ Bemerkungen zu Dietrich von Freiberg, De origine praedicamentalium, in: Von Meister Dietrich zu Meister Eckhart [wie n.18], 34-45; hier p. 44; nochmals das causa-Thema hat Flasch aufgenommen in: Procedere ut imago. Das Hervorgehen des Intellekts aus seinem göttlichen Grund bei Meister Dietrich, Meister Eckhart und Berthold von Moosburg, in: K. Ruh (ed.), Abendländische Mystik im Mittelalter. Symposion Kloster Engelberg 1984, Stuttgart 1986, 125-134.

${ }^{26}$ Gen.I n.4 (LW I, 187 sq.): „metaphysicus rerum entitatem considerans nibil demonstrat per causas extra, puta efficientem et finalem"; Sap.n.20 (LW II, 341).

27 Dietrich von Freiberg, opera omnia, Hamburg 1977, I p. XXIV. 
Jahren) zur Frage der Kompetenz für die Ursachenlehre nur, daß der Begriff der causa in den verschiedenen Disziplinen unter je anderem Aspekt vorkommt: In der Ersten Philosophie wird die causa betrachtet, insofern sie eine disjunkte Bestimmung des Seiendseins darstellt; in der Wissenschaft der Zweiten Analytiken, insofern sie das Prinzip der Erkenntnis des Verursachten ist; und in der Physik, insofern sie in der Weise der Bewegung ein Ding hervorbringt ${ }^{28}$. In seinem Metaphysik-Kommentar aus der Mitte der 1260 er Jahre allerdings findet sich bereits eine Kritik an einem Konzept, in dem - wenn auch in anderer Gruppierung - Physik und Metaphysik durch unterschiedliche causae definiert werden. Im ersten Traktat zum elften Buch heißt es:

„nec etiam est dicendum, sicut quidam alii dixerunt, quod physicus consideret efficientem et materiam et metaphysicus formam et finem ... tamen tam physicus quam metaphysicus considerant omnes quattuor causas" 29 .

Der Editor Bernhard Geyer hat die Anonymisierungsformel "quidam" leider nicht aufgelöst. Immerhin aber findet sich im späteren MetaphysikKommentar, und zwar ebenfalls zum XI. Buch, des Thomas von Aquin eine der Sache nach sehr ähnliche Bemerkung; die Metaphysik, so sagt er, "considerat dicta genera causarum, et praecipue causam formalem et finalem " ${ }^{30}$. Es gab also auch schon vor Dietrich andere Versuche, Metaphysik und Physik durch ihren jeweiligen Begriff von Ursache zu scheiden, nur nicht im Sinne von äußeren und inneren Ursachen - eine Unterscheidung, die Thomas und andere gleichwohl kennen ${ }^{31}$.

2. Die Einschränkung des Ursachebegriffs auf die äußeren Ursachen bleibt zunächst für die Fortentwicklung des Metaphysikbegriffes relativ folgenlos. Nichts mit dieser Zusammenstellung von causa efficiens und causa finalis hat Ockhams Lehre zu tun, die besagt, Wissen als Wissen habe nur diese beiden Ursachen (übrigens als causae essentiales bezeichnet!); als Akzidens der Seele fehlt ihm Materie im eigentlichen Sinne und deshalb auch eine Form ${ }^{32}$. Meister Eckhart übernimmt zwar, wie eben schon gesagt, jene Einschränkung des Ursachebegriffs ${ }^{33}$; nur gehört das Problem, wie sich die Wissenschaften einteilen lassen, gewiß nicht zu seinen primären Interessen. Jener Dietrich'sche Begriff von Ursache wird jedoch hochgra-

28 Phys. II 2, 1 (ed. Col.IV 1 col.97 a).

${ }^{29}$ Met. XI, 1, 3 (ed. Col.XVI 1, 462 sq.).

${ }^{30}$ Met. XI, 1 (2157).

31 S.theol. I-II, 18, 4 ad 2; ScG II, 31 (1081); cf. Philipp der Kanzler, Summa de bono, II q. 2 (ed. N. Wicki, I, 320); Robert Grosseteste, in Post.Anal.II, 2 (ed. Rossi 330).

${ }^{32}$ Expos. Phys., prol. (OPh IV, 7 sq.).

33 Sermo n.511 (LW IV, 425 sq.): ,imago proprie est emanatio simplex, formalis transfusiva totius essentiae purae nudae, qualem considerat metaphysicus circumscripto efficiente et fine, sub quibus cadunt naturae in consideratione physici“". Prinzip und Zweck sind außerhalb: Joh.n.720 (LW III, 630). 
dig wirksam in Eckharts Umsetzung dieses causa-Begriffs in seiner Ethik bzw. seiner spirituellen Protreptik. (Solche Konzepte sind offenbar nicht notwendig an das Problemfeld gebunden, in dem sie entwickelt werden.) Die Transformation zeigt sich nun darin, daß nach Eckhart eine Handlung im strengen Sinne nur insofern eine sittliche genannt werden kann, als deren Zweck mit ihrem Inhalt koinzidiert. Was immer die äußeren Anstöße für ein Handeln sein mögen, es ist doch nur insofern ein sittliches, als diese äußeren Ursachen nicht das Sein des Handelns bestimmen ${ }^{34}$. Es ist also insofern „ohne Warum“. Eckhart übernimmt zwar terminologisch die aristotelische Substantivierung des Interrogativpronomens, schränkt jedoch den Sinn des „Warum“, aus dessen innerer Differenzierung Aristoteles wie gesagt seine Ursachenlehre entwickelt hatte, auf die beiden äußeren Ursachen ein. Da jedoch - ebenfalls gut aristotelisch - Leben als dasjenige gedacht wird, das den Grund seiner Bewegung ,in sich“ (!) hat ${ }^{35}$, kann Eckhart nicht bloß die biblische Redeweise von sittlicher und religiöser Defizienz als „Tod“ interpretieren, sondern das sittliche Leben und das Lebendigsein als solches als eine Strukturidentität denken und dies zuletzt als göttliches Leben vorstellen, denn auch Gott ist ohne die äußeren Ursachen der Finalität und Wirkursächlichkeit tätig ${ }^{36}$. Wenn die Kurzformel dafür lautet: ohne Warum, sine quare, dann heißt dies, daß Eckhart „Ursache“ als etwas Äußeres denkt. Daher trifft sie in diesem Sinn auf das erste Prinzip nicht nur nicht zu, sie ist ihm prinzipiell nicht angemessen.

Von daher liegt es wohl auf der Hand, daß ein berühmt gewordene Vers des Angelus Silesius: „Die Ros blüht ohn Warum; sie blühet weil sie blühet " ${ }^{37}$ nicht bloß innerhalb der Eckhartschen Analogie von Leben und gutem Leben bleibt, sondern dies auch nur deshalb vermag, weil Angelus Silesius jene Neukonzeption der Ursachenlehre unangetastet läßt. Heidegger hat in seiner letzten Vorlesung über den „Satz vom Grund“ diesen silesianischen Vers für eine Einstellung in Anspruch genommen, welche einer Metaphysik entgeht, die sich zur Anerkennung des Prinzips des zureichenden Grundes radikalisiert; dadurch werde an ihr aber nur erst jetzt manifest, was sie je schon war ${ }^{38}$. Ich muß es mir leider versagen, hicr genauer zu verfolgen, wie sich jene angedeutete Rezeptionsgeschichte zu Heideggers Inanspruchnahme des silesianischen Verses im einzelnen verhält. Immerhin sollte sich gezeigt haben, daß der Gedanke des „ohne Warum" selbst zuletzt der Metaphysik und ihrer Reflexion auf die für sie spezifische Begründungsform entstammt.

34 Pred. 4 (DW I, 66); Pred. 39 (DW II, 259 sq.).

35 De an. II, 1; 412 b $15-17$.

36 $\mathrm{BgT}$ (DW V, 43 sq.); cf. R. Schönberger, Secundum rationem esse. Zur Ontologisierung der Ethik bei Meister Eckhart, in: Oikeiosis. Festschrift für Robert Spaemann, ed. R. Iöw, Weinheim 1987, 251-272, hier 270 sq.

37 Cherubinischer Wandersmann, I, 289.

38 Der Satz vom Grund, Tübingen ${ }^{5} 1978$, 68 sqq. 
3. Trotz der internen Zusammenschließung der vier Ursachen ist Dietrichs Gedanke, die Komplementaritätskonzeption als Basis für eine Trennung von Disziplinen zu benutzen, nicht die einzige Folgerung daraus geblieben. Jene Ursachenlehre hat sich auf seine Weise auch Johannes Duns Scotus zunutze gemacht. Gerade Duns Scotus hat bekanntlich einen der wirkungsgeschichtlich bedeutsamsten Schritte zu einer definitiven Trennung von Metaphysik und Physik getan, eine Trennung, die sich in einer Hinsicht ebenfalls an der Ursachenlehre festmachen läßt.

Einer der Anlässe hierfür ist, daß Scotus im „Tractatus de primo principio“ den Status der Begründungen dafür anzugeben hat, daß ein ens infinitum existiert und zwar notwendig existiert; diese Notwendigkeit im Resultat schließt es jedoch aus, von bloß „physikalischen“ Fakten wie dem Gegebensein von Bewegung auszugehen. Für die terminologische Abhebung von Physik und Metaphysik greift Scotus nun ebenso auf die Unterscheidung von causa extrinseca und causa intrinseca zurück. Allerdings interpretiert er ihr Rangverhältnis gerade umgekehrt wie Dietrich von Freiberg. Auch Scotus kommt es zunächst auf die strikte Zuordnung der beiden äußeren und der beiden inneren Ursachen an. Er geht von dieser Zuordnung nicht aus, sondern sucht diese zwingend zu erweisen. Scotus behauptet: Etwas kann nur zielbestimmt sein, wenn es auch bewirkt ist (eine übrigens nicht unwichtige Korrektur an landläufigen Vorstellungen von vorneuzeitlicher Teleologie). Dies Voraussetzungsverhältnis gilt aber auch umgekehrt. Streng analog dazu ist die Struktur bei den inneren Ursachen gebaut. Hinsichtlich des Vollkommenheitsniveaus sind die beiden Ursachearten jedoch ungleich. Mit den äußeren Ursachen ist keine Unvollkommenheit verbunden, wohl aber mit den beiden inneren - und zwar unvermeidlich ${ }^{39}$. Der Grund hierfür ist ein ganz simpler: Die innere Ursächlichkeit von Form und Stoff kann als solche nur fungieren, wenn sie zugleich gewissermaßen zu Teilen eines Ganzen werden. Dies schließt ein Verhältnis wechselseitiger Bedingtheit ein, welches seinerseits wiederum ausschließt, daß die innere Ursächlichkeit zu einem Kandidaten für schlechthinnige Universalisierung werden könnte. Es ist deshalb unmöglich, den inneren Ursachen den Status von „reinen“, soll heißen nicht mehr innerspezifischen Vollkommenheiten zuzuschreiben. Darüberhinaus sind die inneren Ursachen ihrerseits abhängig davon, daß die äußeren Ursachen in Funktion treten. Ohne ein final bestimmtes agens vermag keine Form eine Materie zu formieren. Aus dieser allgemein anerkannten Zuordnung der causae, der sich auch, wie vorher referiert, Dietrich von Freiberg anschließt, zieht Scotus also genau die entgegengesetzten Folgerungen hinsichtlich ihres Status.

${ }^{39}$ Tract. de pr. princ. II n.15 (Kluxen, 18). 
Um etwaigen Zweifeln an der Echtheit des Traktates zu entgehen ${ }^{40}$, sei der Gedanke nochmals im Zusammenhang aus der Ordinatio zitiert:

„cuius non est causa extrinseca, nec intrinseca, quia causalitas causae extrinsecae dicit perfectionem sine imperfectione, causalitas vero causae intrinsecae necessario dicit imperfectionem annexam, quia causa intrinseca est pars causati; igitur ratio causae extrinsecae est naturaliter prior ratione causae intrinsecae ...

Probantur etiam eaedem consequentiae, quia causae intrinsecae sunt causatae ab extrinsecis vel secundum esse earum vel inquantum causant compositum, vel utroque modo, quia causae intrinsecae non se ipsis sine agente constituunt compositum" "41.

Die vier Ursachen betreffen zunächst die Verhältnisse der Bewegung und Veränderung, doch nicht in einem exklusiven Sinne. Als metaphysische causae abstrahieren sie, wie Duns Scotus sich ausdrückt, von sich selbst: „et sic abstrabunt a seipsis ut pertinent ad considerationem physici“" ${ }^{42}$. Was Duns Scotus hier eine Abstraktion nennt, heißt bei Dietrich eine nicht-eindeutige, sondern analoge Gemeinschaft. In beiden Fällen handelt es sich jedoch um eine Ermöglichung von höherer Universalität als es die Ursachen von natürlichen Werdeprozessen erreichen können.

\section{V.}

Die Ausdifferenzierung des aristotelischen Begriffs der Ursache ergibt nicht als solche eine nachträgliche Neuansetzung der Metaphysik, vielmehr wird jetzt die Metaphysik als diejenige Disziplin gefaßt, die ihrem eigenen Anspruch als Form des allgemeinsten Wissens genügt und daher Gründe in ihrer allgemeinsten Form anführt. Was allerdings die Problemlage tiefgreifend verändert hat, war der Umstand, daß es eine gegenüber Aristoteles neue Dimension an Allgemeinheit zu berücksichtigen galt. Der Anspruch, eine unüberbietbare Allgemeinheit des Bewirktwerdens zu denken, erwächst nämlich nun auch, wie unschwer zu sehen ist, aus dem Gedanken der Schöpfung. Dies jedoch in mehrerer Hinsicht: Zunächst in dem Sinne, daß alles, was nicht Gott ist, durch ihn ist, wie Augustinus sagt und seither oftmals wiederholt worden ist ${ }^{43}$. Dann aber auch in einem

40 Cf. V. Richter, Studien zum literarischen Werk von Johannes Duns Scotus, München 1988.

41 Ord.I d.2 p.1 q.1-2 n.57 (ed. Vat.II, 163 sq.); dieselbe, aber stark verkürzte Argumentation mit dem Begriff des Teilseins in Rep.Par.I d.2 q.2 n.3 (XII, 64 a); n.6 (65 b): ,esse effectivum nullam imperfectionem importat de se".

42 Tract. II n.12 (Kluxen, 14).

43 Augustinus, De Trinitate I 6, 9 (CCSL 50, 38): „Omnis enim substantia quae Deus non est creatura est, et quae creatura non est Deus est", Anselm von Canterbury, De casu diaboli, 1 (I p. 233); Thomas von Aquin, ScG II, 15 (922): "nibil praeter ipsum nisi ab ipso"; De aeternitate mundi, ed. Leon. XLIII p. 85 1.10-13: „... philosophos, qui confitentur et probant omne quod est quocumque modo esse non posse, nisi sit causatum ab eo qui maxime et verissime esse babet". Für Buridan ist dies ein Argument gegen die Lehre vom complexe significabile: Soph. I, 5 (Scatt 24). 
Sinne, der es unmöglich macht, den creator als einen, wenn auch vielleicht höchsten Fall der aristotelischen causa efficiens zu fassen. Daß hier eine Unmöglichkeit vorliegt, zeigt sich schon daran, daß der uns so geläufig und selbstverständlich gewordene Ausdruck causa efficiens zwar ein scholastischer, nicht aber der aristotelische ist. Wenn Aristoteles von derjenigen Ursache spricht, die etwas hervorbringt, nennt er sie durchweg ő $\theta \varepsilon v \grave{n}$

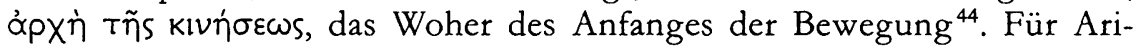
stoteles besagt die sog. Wirkursache soviel wie Bewegungsursache; wie immer über die ursächliche Funktion der platonischen Ideen zu denken ist, sie sind für Aristoteles insbesondere deswegen nicht hinreichend, weil sie für Veränderung nicht aufkommen können; die Idee der Gesundheit als solche macht nicht gesund. Wenn dagegen nun der Sinn von Grund die Bewegung miteinschließen muß, dann ist der erste Grund ebenfalls ein Bewegendes, aber dadurch und darin ein erster, daß er unbewegt ist. $\mathrm{Da}$ jedoch, so kann man auch Aristoteles-immanent einwenden, das Unbewegt-sein auch ein Prädikat inner-kosmischer Instanzen sein kann, kann einerseits das Unbewegte nicht als etwas angesetzt werden, das der ursächlichen Begründung nicht bedürftig wäre, und andererseits Bewegung nicht als die universelle Weise der Hervorbringung verstanden werden.

Hier war es wohl Etienne Gilson, der als erster darauf aufmerksam gemacht hat ${ }^{45}$, daß Albertus Magnus ohne Zögern, aber auch ohne den Schritt in seiner grundsätzlichen Bedeutung eigens hervorzuheben, von fünf Ursachen spricht: "quidam dixerunt causas esse quinque" ${ }^{46}$. Gemeint ist damit eindeutig nicht etwa eine aus Konkordanzgründen integrierte causa exemplaris - die übrigens später, bei Duns Scotus in die causa efficiens ${ }^{47}$ und bei Buridan in die causa formalis zurückgenommen wurde -, sondern eine interne Aufspaltung der Wirkursächlichkeit selbst. Diese hat in weiten Kreisen eine Fortsetzung gefunden. Man findet diese Aufspaltung bei Siger von Brabant, bei Petrus von Alvernia etc. Analog zur lateinisch gefaßten aristotelischen Formel der Wirkursächlichkeit als unde principium

${ }^{44}$ Met. I, 2; 983a30; c.4; 985a13 c.7; 988a33; c.9; 991a26; V, 2; 1013b23-25; etc. - W. Wieland hat im (n.5) genannten Buch auch auf den Umstand aufmerksam gemacht, daß Aristoteles ,immer nur ein Ding auf seine Ursachen hin befragt, niemals aber im Ausgang von gegebenen Ursachen nach,Wirkungen' [ge]forscht. Man sieht das schon daran, daß ein der modernen ,Wirkung" entsprechender Grundbegriff in der aristotelischen Pbysik feblt." (266) Es besteht also weder eine eineindeutige Relation zwischen Ursache und Verursachtem, noch stehen sie überhaupt „auf derselben begrifflichen Ebene“, die eine ist die von Dingen, die andere die von Prinzipien.

${ }^{45}$ Notes pour l'histoire de la cause efficiente, in: AHDLMA 37 (1962), 7-31; mehrfach weitergeführt bei W. Dunphy, Peter of Auvergne and the twofold efficient cause, in: Med.Stud.28 (1966), 1-21; id., Albert and the five causes, in: AHDLMA 41 (1966), $7-$ 21.

${ }^{46}$ Met. V, 1, 3 (ed. Col. XVI,1 p. 214, 41-42).

47 Ord.I d.36 q. un. n.23 (ed. Vat.VI, 280): „causa exemplaris non est nisi quoddam efficiens"; Ockham, ord.I d.35 q. 5 (OTh IV, 501): "idea vel exemplar non est proprie causa“. 
motus sprechen diese von einem unde principium esse. Dies geht zuletzt wohl auf Avicenna zurück; den Text werde ich noch zitieren. Wichtig ist für unsere Fragestellung, daß auch diese innere Dissoziierung der Wirkursächlichkeit mit dazu dient, die Disziplinen des theoretischen Denkens voneinander abzugrenzen; bei Petrus von Alvernia heißt es: „primum efficiens usitatur in naturalibus, secundum autem in mathematicis et divinis" ${ }^{8}$. Die Bewegungsursächlichkeit der Naturphilosophie und die Seinsursächlichkeit der Ersten Philosophie zuzuschreiben scheint ein so zwingender Gedanke zu sein, daß er auch jenseits von Schulgrenzen akzeptiert wird; trotz aller Variationen läßt er sich bei sonst so verschiedenen Denkergestalten wie Ulrich von Straßburg ${ }^{49}$, Heinrich von Gent ${ }^{50}$ und selbst bei Johannes Buridan ${ }^{51}$ belegen.

Erst jetzt ist es einer theologischen Wissenschaft möglich, Schöpfung nicht als einen halb-mythologischen Prozeß zu denken; sowohl Thomas ${ }^{52}$ wie Duns Scotus ${ }^{53}$ - und übrigens auch der Savoyardische Vikar ${ }^{54}$ haben nämlich bestritten, daß Schöpfung eine Veränderung und mithin eine Bewegung sei. Thomas allerdings fügt diese ausdifferenzierte Seinskausalität in eine umfassendere Seinslehre ein. Insbesondere in den Kapiteln 15 und 16 des zweiten Buches der „Summa contra gentiles“ widmet er diesem Versuch eine ausgedehnte Argumentation. An dieser wird merklich, $\mathrm{da} ß$ es sich nicht bloß um eine Absetzbewegung vom Bewegungspara-

48 In Met. III, 3 (ed. W. Dunphy, in: Med.Stud.26, 1964, 291).

49 De summo bono II, 2, 2 (CPTMA I, 2.1 p. 30 sqq.).

50 Sum. 24, $6(141 \mathrm{rN})$; dort wird die Rangordnung zwar nicht ausdrücklich gemacht, aber offenkundig deswegen, weil sie schon bei ihm selbstverständlich ist; cf. Sum.22, 5 (135rEF); ganz ähnliche Einschätzung bei E. Gilson, History of Christian Philosophy in the Middle Ages, London 1955, 448. cf. qdl.1, 7-8 (V, 37): „cum omnis transitus factionis de non esse sit transmutatio et haec transmutatio naturalis quando est circa subiectum praeexistens, actus creationis, etsi non sit vera transmutatio ut est illa quae est naturalis, quia tamen est de non esse in esse, modum mutationis habet, non motus, ad modum actionis qua rei acquiritur esse per generationem naturalem." Von diesen Quaestionen aus könnte auch ein Licht auf die Motive für die Verurteilung des Satzes fallen: "Quod creatio non debet dici mutatio ad esse ..." (217) Eine mutationslose Hervorbringung gilt von den trinitarischen Prozessen, nicht aber von der Erschaffung; sie würden also ununterscheidbar.

51 Met. I, 4 (5va): ,nec valet etiam quod dicebatur, scilicet quod physica etiam considerat primam causam. Dico quod verum est, licet hoc non est modo quidditativo, immo solum respective ad motum et iste modus considerandi est posterior, quia est respectivus ad posteriora. Metapbysica autem modo priori scilicet quidditative considerat Deum et intelligentias [Ed.: intelligendas]. Ideo ipsa metaphysica est simpliciter prima. IV, 3 (14vb): „physicus considerat de omnibus entibus, non quomodo sunt, sed quomodo movent".

52 Cf. n.23; ScG II, 37 (1131); Sum.theol.I, 45, 2 ad 2; ad 3; 45, 3; 46, 3 ad 2; de pot.3, 2.

53 Rep.Par. I d.1 q.6 n.22 (ed. Vivès XXII, 558 a). - zu Bonaventura, Sent. II p.1 a.1 q.2 ad 1 (II, 23 b): „loquendo autem de mutatione supernaturali, per quam ipsum mobile processit in esse ..."; Sent. II d.1 p.1 a.3 q.1 (II, 32 b); E. Gilson, La philosophie de Saint Bonaventure, Paris ${ }^{3} 1953,164$.

54 Profession de foi du vicaire savoyard, 1914, 255; cf. H. Deku, De nihilo [1974], in: Wahrheit und Unwahrheit der Tradition, St. Ottilien 1986, 224 sq. 
digma des Aristoteles handelt, sondern natürlich auch um eine Auseinandersetzung mit denjenigen, die meinen, auf eine solche Distanzierung verzichten zu können ${ }^{55}$.

Ich muß es mir hier versagen, die Argumente des Thomas im einzelnen zu erläutern und zu diskutieren, in denen er diesen Gedanken einsichtig zu machen sucht. Nur soviel: Die erste Ursache kann solange nicht als eine universelle gedacht werden, als sie lediglich als Bewegungsprinzip, malitiös gesagt als Kosmosmotor gedacht wird. Begründungsbedürftig ist aber nicht erst das bloße Bewegtsein, sondern schon, daß etwas ist. Thomas sagt ausdrücklich: „esse autem est universalius quam mover ${ }^{\text {« } 56}$ und das soll zugleich heißen, es ist auch nichts allgemeiner als das Seiendsein. Wenn der kreativen Hervorbringung des ersten Prinzips aber schlechthin alles unterworfen sein muß, so ergibt doch auch die Reflexion auf das Phänomen der Bewegung selbst, daß es das zu Bewegende bereits voraussetzt. Ein erstes Prinzip ist daher kein bloßer Beweger (936). Die Urhebung von Sein läßt sich nicht denken als Veränderung des Nichtseins. Diese damit zusammenhängende Unterscheidung von causa essendi und causa fiendi könnte man auch weit außerhalb der thomistischen Schulrichtung vielfach belegen und kehrt mit den alten Schulbeispielen sogar noch bei Descartes wieder.

Merkwürdig bleibt freilich, daß im wohl berühmtesten Text der Scholastik, den quinque viae, dieses Gesichtspunkt völlig ausgespart bleibt. Dort heißt es, der Ansatz ex motu sei der eindrücklichste und selbst ein Heinrich von Gent wird dies wiederholen ${ }^{57}$. Von der Reflexionsstufe des contragentiles-Kapitels her wird es noch problematischer, vom primum movens immotum zu sagen, ,et hoc dicimus omnes Deum“. Das Unbewegtsein ist für

${ }^{55}$ Vom Sentenzen-Kommentar (Sent. II d.1, 1, 5 ad 1: "sicut dicit Commentator in lib. De substantia orbis, cap. 2, Aristoteles nunquam intendit quod Deus esset causa motus caeli tantum, sed etiam quod esset causa substantiae eius, dans sibi esse.") bis zu den späten AristotelesKommentaren hat Thomas daran festgehalten, daß es nicht der Meinung des Aristoteles entspricht, die Begründungskompetenz des ersten Prinzips bloß auf die Bewegung einzuschränken: in Phys.VIII, 3 (996); VIII, 21 (1154); in Met. II, 2 (295); VI, 1 (1164); in de cael.I, 8 (91).

${ }^{56}$ ScG II, 16 (935); in de causis 18 (342): „moveri praesupponit esse“; weit strikter Duns Scotus, ord.II d.1 q.3 n.115 (ed. Vat. VII, 57): „efficiens in plus est quam movens; dazu allerdings n.153 (p. 77); Met. I q.1 n.25 (ed. Vivès VII, 23 a): „quatuor causae, inquantum quaelibet in suo genere dat esse circumscribendo rationem motus et mutationis, pertinet ad Metaphysicum; materia et forma, inquantum sunt partes essentiae; efficiens, inquantum dat esse circumscibendo motum, licet enim non ageret nisi movendo, tamen ratio dantis esse, prior est ratione moventis ..." cf. E. Gilson, Jean Duns Scot, Paris 1952, 82 n.1: „Ne se plaçant pas, comme avait fait Thomas d'Aquin, au point de vue de l'existence même du mouvement, Duns Scot a le droit d'admettre que le ,premier moteur' n'est pas nécessairement le ,premier être'.“

57 Sum.theol.I, 2, 3; in Phys.VIII,1 (970); Heinrich, Sum.22, 4 (132vM): „... et sunt tres in via causae efficientis. Quarum prima et manifestior est illa ..." 
sich allein genommen gerade nicht dazu hinreichend, sich als Begriff eines schlechthin Ersten zu prädestinieren ${ }^{58}$.

Zwei Dinge sind hier allerdings zu beachten: Zum einen liegt darin nicht eine Inanspruchnahme des Ursachebegriffs über die naturalen, also quoad nos zunächst begründungsbedürftigen Verhältnisse, sondern es wird die schon neuplatonische Behauptung fortgeführt, das erste Prinzip sei sogar im eigentlichsten Sinne Ursache ${ }^{59}$. Zum anderen aber wird dieser Status als eines Inbegriffs neu interpretiert: Die theologische Richtung denkt Gott selbst als Grund von Ursächlichkeit, als causa causalitatis. Thomas von Aquin integriert dies in den Gedanken, daß das ens als solches eines Grundes bedürftig sei und dieser im ipsum esse subsistens liege. Vor dem Hintergrund der eingangs angestellten Überlegungen zur aristotelischen Lehre von den vier Ursachen kann man also gerade an diesem Kernbestand des Aristotelismus die Transformationskraft der Scholastik eindrücklich machen.

Ein solches Vorgehen ergibt sich zum einen aus einem Konkordanzinteresse: Durch die Distinktion wird ein Gedanke eingeführt, den Aristoteles als solchen weder affirmieren noch bestreiten konnte; in seiner partikularen Geltungsform braucht er jedoch vom Exegeten auch nicht zurückgewiesen zu werden. Diese gedankliche Bewegung nimmt sich wie ein Fortschritt in der Wissenschaft: Aus einer universelleren Perspektive wird eine andere sowohl als partikulare erkannt, wie dann auch in die universellere integriert.

Allerdings ist diese Theorie der Verursachung von Seiendsein nicht unwidersprochen geblieben. Zwei Kritiker und Kritikpunkte seien genannt:

Siger von Brabant verwirft völlig den Gedanken einer Verursachung von Seiendsein. In seinem Metaphysik-Kommentar bringt Siger die unvermeidlich an Leibniz erinnernde - Frage auf, warum überhaupt etwas ist und nicht vielmehr nichts: „quare magis est aliquid in rerum natura quam nibil?" ${ }^{60}$. Interessant daran ist weniger die vielleicht kuriose Vorwegnahme, als vielmehr der Umstand, daß Siger die Frage als solche für sinnlos hält. So wie sie gestellt ist, kann sie als Antwort nur selbst wieder ein Seiendes anzielen, die Frage läßt sich also in dieser Allgemeinheit gar nicht stellen. Die Warum-Frage setzt somit ein zumindest in irgendeiner Hinsicht spezifiziertes Seiendes voraus. Bekanntlich hat Thomas von Aquin die Frage nach dem Grund des Seiendseins nicht nur für sinnvoll gehalten, sondern

58 Wenn man diese Texte gegeneinanderhält, fällt auf die thomistischen und neuscholastischen Rehabilitierungsversuche der prima via ein seltsames Licht; ob der Beweisgang argumentationstheoretisch vergeblich ist, dies zu untersuchen ist nicht mein Thema; jene Rehabilitierungsversuche haben jedoch nur zu oft kein Verhältnis zu der zuletzt genannten Perspektive des Thomas.

59 Auch schon Ps.-Platon, Epinomis 981 a: åpxń Tìs åpxர́s.

(x) In Met. IV, comm.; ed. Dunphy, 170; cf. Introd. q. 2 (Dunphy, 37). 
als definitorische Bestimmung des Gegenstandes der Metaphysik angeführt; bezeichnenderweise aber nur deshalb, weil dieser Grund selbst gerade nicht als ein Seiendes gedacht wird. In anderer Weise wird diese wohl „ontologisch“ zu nennende Differenz bei Meister Eckhart angesetzt, wenn er als Grund des Seienden das reine Nichts zu denken versucht. Es ist deutlich, daß Leibniz sich von beiden Konzeptionen unterscheidet.

Wenn auch die an Leibniz gemahnende Formulierung Sigers zu verlokkend ist, um sie hier zu übergehen, muß doch der historischen Angemessenheit wegen gesagt werden, daß Sigers Ablehnung keine singuläre Ausnahme bildet. Ähnlich argumentieren nämlich beispielsweise Heinrich von Gent ${ }^{61}$ und Duns Scotus ${ }^{62}$. In allen Fällen ist es dasselbe Argument wie schon bei Avicenna: „Deinde principium non est principium omnium entium. Si enim omnium entium esset principium, tunc esset principium sui ipsius; ens autem in se absolute non babet principium; sed babet principium unumquodque esse quod scitur"63. Die Universalierung des Begriffes von Grund führt auf den widerspruchsvollen Begriff der causa sui. Nur in Parenthese sei dazu angemerkt: Von dieser Argumentation her kann man verstehen, warum im 17. Jahrhundert, wo das Prinzip des zureichenden Grundes, welches als universell geltendes Prinzip für alles Seiende schlechthin einen Grund fordert, explizit formuliert wurde, dies überall die Verwendung des Begriffes causa sui nach sich gezogen hat. Schon Avicenna hat gesehen, daß hier ein Implikationsverhältnis vorliegt. Nur war man jetzt bereit, den vormals widersprüchlich scheinenden Begriff der causa sui zu verwenden. Dies gilt sowohl für Spinoza wie für Descartes; aber auch für Leibniz. Hatte sich dieser in seinen Anmerkungen zur Ethik des Spinoza eher zurückhaltend gezeigt, so finden sich anderwärts auch bei ihm durchaus Ausdrucksweisen, die den Gedanken der causa sui enthalten.

Zurück zum scholastischen Zusammenhang. Der im Kontext des Unmöglichkeitsargumentes erwähnte Duns Scotus hat auch noch ein anderes spezifisches Argument vorgebracht: Der Gedanke einer causa essendi universalis mache zuletzt doch die causae secundae redundant. Wenn nicht diese Sein hervorbringen, sondern lediglich die causa prima, so fragt sich, welche kausale Funktion den causae secundae noch verbleibt. Bekanntlich hat Thomas der Begründung endlicher Kausalität seine besondere Aufmerksamkeit gewidmet. Das Etwas-hervorbringen-können, das Wirksam-werden-kön-

61 Sum.quaest. 6, 2 ad $4(115 \mathrm{vL})$ : "prima philosophia, quae est de ente simpliciter ut de subiecto, non considerat principia aliqua subiecti, quia non babet ulla. Ens enim simpliciter ... non babet principium, sed est principium aliquibus entibus."

62 Ord.I prol. p. 3 q.1 - 3 n.191 (ed. Vat. I, 128): „entis inquantum ens, quod ponitur subiectum metaphysicae, nulla sunt principia, quia tunc essent cuiuslibet entis"; so auch Suarez, Disp. met.I 5, 38 (XXV, col.48a).

${ }_{6.3}$ Met. I, 2 (Van Riet, 14); auf die zuletzt zitierten Stellen hat bereits Albert Zimmermann aufmerksam gemacht: Die "Grundfrage" in der Metaphysik des Mittelalters, in: Arch. Gesch. Philos. 47 (1967), 141-156. 
nen ist für ihn das stärkste Indiz für Wirklichkeit überhaupt ${ }^{64}$. Zwar bestreitet Thomas nicht - wie manche der Artes-Lehrer ${ }^{65}$-, daß causae secundae aufgrund ihres instrumentellen Status in der unmittelbaren Wirksamkeit der causa prima ersetzt werden können; dies hat jedoch lediglich die Form eines Zugeständnisses, denn die dignitas causalitatis, die Würde, etwas hervorbringen zu können - ein Ausdruck, den Thomas mehrfach verwendet ${ }^{66}$ (und übrigens auch noch Pascal) - gehört selbst zum Gehalt der Schöpfung. Die Ersetzbarkeit ist daher lediglich eine supranaturale Möglichkeit, nicht etwas, das aus Gründen der Ökonomie genutzt würde!

Ockham wird freilich die Frage stellen, ob wir darüber Gewißheit gewinnen können, wann ein Fall natürlicher Kausalität und wann deren supranaturale Ersetzung vorliegt. Seine Antwort ist, daß es darüber bei natürlichen Ursachen keine absolute Gewißheit geben kann; ohne davon besonderes Aufheben zu machen, nimmt Ockham allerdings den Willen davon aus: Mein Wollen ist zwar eine sekundäre Ursache, jedoch nicht in einem sittlich relevanten Sinne ersetzbar, auch nicht durch den Schöpfer des Willens: "omnis actus alius a voluntate potest fieri a solo Deo ..." 67 .

Läßt sich aus all dem am Ende noch ein philosophisches Resümee ziehen? Die verschiedenen, hier durchgegangenen Versuche, Metaphysik und Physik zu differenzieren, sind insgesamt betrachtet nur ein Stadium in der komplexen Geschichte des Verhältnisses von Metaphysik und Physik, das nicht zu einer völligen Scheidung beider Disziplinen führt. War der aristotelische Bewegungsbegriff einer, der nicht allein durch seine antieleatische Intention, sondern auch durch die verwendeten Modalbegriffe offenkundig meta-physisch ist, so ist doch ebenso offenkundig auch bei den hier betrachteten Autoren die qualitative Physik in Kraft geblieben. Diese erhält jedoch einen neuen Status. Insofern eine Scheidung von Physik und Metaphysik unter dem Gesichtspunkt der Kontingenz unternommen wurde, ergibt sich daraus zweierlei: Der Metaphysik wird nicht allein wie auch schon bei Aristoteles - eine andere Ebene zugewiesen, sondern diese Ebene überhaupt wird neu definiert: Sie ist erstens von größerer, ja

${ }^{64}$ ScG III, 69; im cap. 66 hatte es geheißen, daß was immer Sein verleiht, dies in virtute divina tut. Es handelt sich ja nicht um zwei toto coelo verschiedene Leistungen, vielnehr spezifizieren und determinieren die secunda agentia das vom primum agens verliehene Sein: III, 66 (2412).

${ }^{65}$ Siger von Brabant, qu. de causis, q. 2 (ed. A. Marlasca 41); auch in einem anonyınen Physikkommentar (Paris, BN 16 297), der vielleicht von Siger stammt: in Phys.I q. 13 (ed. A. Zimmermann, Ein Kommentar zur Physik des Aristoteles, Berlin 1968, 25); Johannes Wacfeld, in Phys; cod. Gonville and Caius 344, f.271va (cit. ap. Zimmermann, Ein Kommentar, XXXIX); cf. R. Hissette, Enquête sur les 219 articles condamnés à Paris le 7 Mars 1277, Louvain-Paris 1977, 128 sq.; auch ein Dietrich von Freiberg bestreitet die Berechtigung der Berufung auf die prop.1 des L.de causis: de acc.23, 3 sqq. (III, 86 sq.).

6r. Sent. II d.1, 1, 4; sum.theol.I, 23, 8 ad 2.

${ }^{67}$ Qdl. 3, 14 (O'Th IX, 254). 
jetzt sogar von größtmöglicher Universalität und zweitens von höherer und jetzt sogar von grundsätzlicherer Unabhängigkeit von der Physik. Vielleicht darf man so weit gehen zu sagen, hier bahne sich zumindest die Möglichkeit an, die Physik als ein Unternehmen zu betrachten, dessen Resultate unvermeidlich die Züge der Kontingenz tragen. Kontingenz wird also durch Kausalität nicht an sich beseitigt oder bewältigt. Immerhin werden dann schon im 14. Jahrhundert die aristotelischen Notwendigkeitsansprüche für seine Kosmologie (gegen die des Timaios) auf breiter Basis eingezogen ${ }^{68}$. Umgekehrt hat die Metaphysik in dieser Phase die äußerste Anstrengung unternommen, ihre Begründungsform mit dem ihrer Disziplin immanenten Allgemeinheitsanspruch zur Konsistenz zu bringen. Insofern sie dies erreicht, emanzipiert sie sich von einer Naturphilosophie, für die sie nicht nur einmal die spekulativen Voraussetzungen bereitgestellt hat, von der aber auch in dieser Zeit zunehmend durchschaubar wird, daß sie auch andere, nämlich kosmologische Voraussetzungen macht, deren Kontingenz der Selbstbehauptung der Metaphysik eine zusätzliche Selbstreflexion mit neuen Resultaten abverlangt.

${ }^{68}$ Cf. vom Vf., Eigenrecht und Relativität des Natürlichen bei Johannes Buridanus, in: Misc. Med. XXI, 1 (Mensch und Natur im Mittelalter), ed. A. Zimmermann/A. Speer, 1991, 216-233. 